\title{
New Insights into MHD Dynamics of Magnetically Confined Plasmas from Experiments in RFX
}

P. Martin, S. Martini, V. Antoni, L. Apolloni, M. Bagatin, W. Baker, O. Barana, R. Bartiromo,

P. Bettini, A. Boboc, T. Bolzonella, A. Buffa, A. Canton, S. Cappello, L. Carraro, R. Cavazzana, G. Chitarin, S. Costa, F. D’Angelo, S. Dal Bello, A. De Lorenzi, D. Desideri, D. Escande*, L. Fattorini, P. Fiorentin, P. Franz, E. Gaio, L. Garzotti, L. Giudicotti, F. Gnesotto, L. Grando, S.C. Guo, P. Innocente, A. Intravaia, R. Lorenzini, A. Luchetta, G. Malesani, G. Manduchi, G. Marchiori, L. Marrelli, E. Martines, A. Maschio, A. Masiello, F. Milani, M. Moresco, A. Murari, P. Nielsen, M. O’Gorman, S. Ortolani, R. Paccagnella, R. Pasqualotto, B. Pégouriè**, S. Peruzzo, R. Piovan, N. Pomaro, A. Ponno, G. Preti, M.E. Puiatti, G. Rostagni, F. Sattin, P. Scarin, G. Serianni, P. Sonato, E. Spada, G. Spizzo, M. Spolaore, C. Taliercio, G. Telesca, D. Terranova, V. Toigo, L. Tramontin, M. Valisa, N. Vianello, M. Viterbo, L. Zabeo, P. Zaccaria, P. Zanca, B. Zaniol, L. Zanotto, E. Zilli, G. Zollino.

Consorzio RFX, Associazione Euratom - ENEA sulla Fusione, Corso Stati Uniti 4, 35127 Padova, Italy

* UMR 6633 CNRS-Université de Provence, Avenue Normandie-Niemen, 13397 Marseille Cedex 20, France

** Association EURATOM-CEA sur la Fusion Contrôlée, C. E. Cadarache, 13108 Saint-Paullez-Durance, France

e-mail contact of main authors: martin@igi.pd.cnr.it - martini@igi.pd.cnr.it

\begin{abstract}
The experimental and theoretical activity performed in the RFX experiment has allowed a deeper insight into the MHD properties of the RFP configuration. A set of successful experiments has demonstrated the possibility of influencing both the amplitude and the spectrum of the magnetic fluctuations which characterise the RFP configuration. A new regime (QSH states) where the dynamo mechanism works in a nearly laminar way and a helical core plasma is produced has been investigated. With these studies a reduction of the magnetic chaos has been obtained. The continuos rotation of wall locked resistive tearing modes has been obtained by an $m=0$ rotating perturbation. This perturbation induces rotation of $m=1$ non-linearly coupled modes.
\end{abstract}

\section{Introduction}

The search for advanced and innovative confinement scenarios is one of the major challenges in the studies on magnetic confinement of fusion plasmas. An important aspect in this task is the optimisation of magnetic equilibria and the understanding and control of magnetohydrodynamic (MHD) phenomena. A well diagnosed, large Reversed Field Pinch (RFP) experiment like the RFX device (major radius $R=2 \mathrm{~m}$, minor radius $a=0.46 \mathrm{~m}$ ) [1] represents an excellent test bench for many MHD studies, since MHD dynamics displays a variety of features in RFP's.

MHD instabilities are important in the RFP, since they break the toroidal symmetry of the magnetic field and drive the self-generation of the reversed toroidal magnetic field through a mechanism traditionally called dynamo [2]. The RFP equilibrium in cylindrical coordinates is in fact characterised by a safety factor $q(r)=\frac{r B_{\varphi}(r)}{R B_{\vartheta}(r)}$ which is typically close to $a / 2 R$ in the plasma core and decreases to a slightly negative value at the edge. This means that the poloidal field in 
the RFP is of the same order of magnitude as the toroidal field. Moreover, the reversal of the toroidal field at the edge shows that poloidal electric currents must flow in the plasma to generate the positive toroidal field in the centre. These currents cannot be directly driven by the inductive toroidal electric field produced by the transformer effect, and a Lorentz, "dynamolike" contribution $\boldsymbol{v} \times \boldsymbol{B}$ is therefore necessary. This implies the existence of a self-organised velocity field in the plasma, which couples to part of the magnetic field to produce the required electric field.

A series of numerical simulations $[3,4,5]$ and experimental results (see for example $[2,6]$ and the references therein quoted) has shown that a robust mechanism to produce the dynamo electric field is that driven by magnetic field and velocity fluctuations associated to MHD tearing modes. In particular, a recent investigation of the trajectory deflection of frozen hydrogen pellets launched into the RFX plasma [7] has allowed to diagnose the suprathermal electrons in the plasma and, to this end, the electron distribution function. The results of this work indicate that the pellet trajectory can be described using the classical Spitzer-Harm distortion of the Maxwellian distribution function caused by the MHD dynamo electric field. This gives a further confirmation that a dynamo mechanism based on a local mean field electrodynamics theory can account for the observed magnetic field.

Due to the shape of the $q$ profile, many tearing modes, resonant on closely spaced rational surfaces, can indeed be simultaneously destabilised. As a result the instabilities generating the dynamo have, typically, a wide $k$ spectrum. Many $m=0$ and $m=1$ modes with different toroidal mode numbers $n$ 's and similar amplitudes are in fact simultaneously present in the standard Multiple Helicity (MH) RFP state. The global normalised fluctuation amplitude is observed to scale with the magnetic Lundquist number $S$ as $b / B \propto S^{-0.16 \pm 0.02}$ [8], which is in good agreement with the output of numerical simulations [5]. These magnetic fluctuations destroy closed magnetic surfaces in the plasma core and induces magnetic chaos over large portions of the plasma volume. This produces a high transport in the radial direction and therefore spoils particle and energy confinement. Moreover, through phase locking of the modes, a toroidally localised distortion of the magnetic equilibrium and a bulging of the plasma are produced. When modes lock also to the wall in a particular toroidal position, for example because of field errors, this results in a strong plasma-wall interaction [9]. This latter feature, despite the different origin, is shared with other toroidal configurations, as tokamak for example.

The study of the mechanisms driving MHD instabilities and of the techniques to control them is therefore crucial for RFP research. In this paper we describe the main recent theoretical and experimental results on MHD studies from the RFX experiment. In particular, Sect. 2 contains the description of experimental and theoretical results concerning a new self-organised laminar dynamo regime, i.e. the Quasi Single Helicity regime. In Sect.3 we will present the magnetic fluctuations active control technique which have been successfully developed in RFX; conclusions and indications for future work are the subject of Section 4.

\section{Quasi single helicity (QSH) states}

Recent experimental measurements in RFX [10,11,12] have shown that a different dynamo regime can be present, where magnetic field topology might be rather different from that 
observed in the standard, wide-spectrum MH case. In this regime, dubbed Quasi Single Helicity (QSH), the $m=1$ magnetic field fluctuations spatial spectrum is made up almost entirely by one individual $(m=1, n \approx 2 \mathrm{R} / \mathrm{a})$ MHD mode. In this case the symmetry breaking required to have a stationary RFP equilibrium in a resistive plasma is provided by the saturation of a resistive kink mode. This is rather different from both the helical state predicted by Taylor [13] and from the most common and previously discussed $\mathrm{MH}$ state. In this section we present the most important experimental features of QSH states and an outline of the theoretical work for their understanding. A more complete coverage of the QSH theory can be found in [14].

\subsection{Experimental results on QSH states}

QSH states are reproducibly obtained in RFX either transiently (for several ms) or in stationary

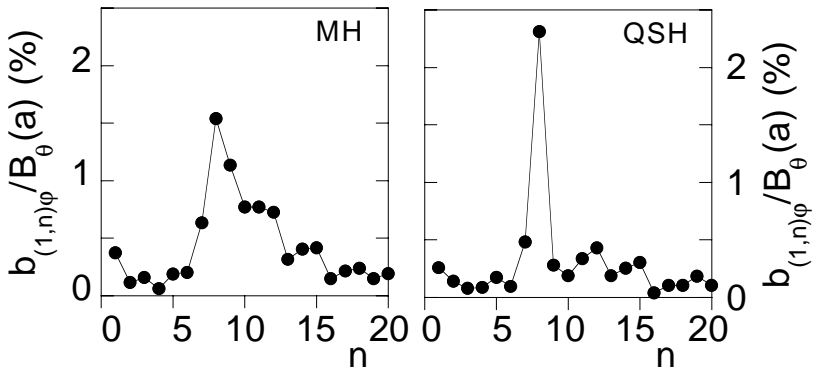

Figure 1: $m=1$ toroidal spectra for a $\mathrm{MH}$ plasma and for a QSH plasma. conditions (i.e. during the whole pulse duration) [11]. These time scales have to be compared with the confinement time $\leq 2 \mathrm{~ms}$ and the discharge duration $\approx 0.1 \mathrm{~s}$. Figure 1 illustrates the difference between a $\mathrm{MH}$ and a QSH plasma in term of MHD modes. The two discharges have similar global parameters, but in the QSH case it is evident the predominance of the $(m=1, n=8)$ mode. On the contrary all the $m=1$ modes between $n=7$ and $n=12$ have comparable amplitude, within a factor of two, in the $\mathrm{MH}$ case. To quantify the difference between the two spectra it is convenient to introduce a measurement of the "width" in the toroidal mode number $n$ axis of the $m=1$ spectrum. This is done using the spectral spread, $N_{\mathrm{s}}$ defined as $N_{s}=\left[\sum_{n}\left(W_{n \varphi} / \Sigma_{n} W_{n \varphi}\right)^{2}\right]^{-1}[15]$, where $W_{n, \phi}$ is the energy of the $(m=1, n)$ mode. $N_{s}$ is an effective indicator of the number of $n$ modes composing the spectrum. A pure Single Helicity spectrum has $N_{\mathrm{S}}=1$. For the cases shown in Figure 1 we have $N_{\mathrm{S}} \approx 3.7$ for the $\mathrm{MH}$ plasma and $N_{\mathrm{S}} \approx 1.2$ for the QSH state.

The exploration of the plasma core reveals that a different magnetic topology is associated to QSH states. An imaging of the plasma core can be obtained through soft x-ray (SXR) tomography [12], since SXR iso-emissive surfaces can in fact be considered as representative of magnetic surfaces in the MHD framework. Figure 2 shows the images of the SXR emissivity taken in a MH and in a QSH plasma.. A poloidally symmetric emissivity is found in the MH state. In contrast a "bean"-like hot $m=1$ structure is evident in the QSH case. The location of the island systematically coincides with the resonance radius and poloidal phase angle of the dominant $m=1$ mode, which are reconstructed from magnetic measurements. This evidence suggest that helically symmetric closed magnetic surfaces are generated. The improvement of the magnetic flux surfaces in the plasma core is hinted also by direct electron temperature and density profile measurements, which indicate that this helical structure confines a higher 
pressure than the plasma nearby $[10,12]$. Indeed helical hot regions where the electron temperature can be $>50 \%$ higher than the neighbouring bulk plasma are systematically observed in RFX during QSH states both by multipoint Thomson scattering and by SXR tomography. As discussed in the next section and in [14], the heating of the helical structure has been explained in terms of an increased resilience to chaotic perturbations when the island separatrix vanishes.

The onset of a QSH state has relevant consequences not only for the plasma core, but also for the edge and for the plasma-wall interaction [16], since we observe a reduction of the localised bulging of the plasma column.
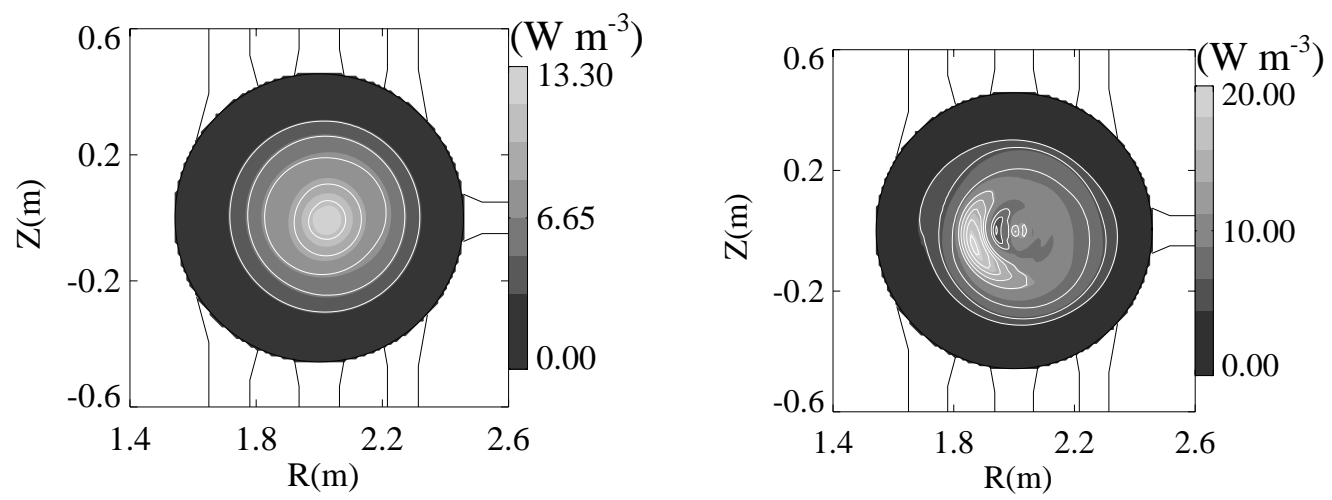

Figure 2: SXR emissivity for a MH and a QSH plasma

\subsection{Theoretical aspects of single helicity}

The recent experimental results on QSH motivated a thorough theoretical study of the single helicity (SH) states of the RFP which led in particular to the two following results $[17,18]$. A scaling approach to the simplest visco-resistive MHD model reveals that the Prandtl number acts only through the inertia term. When this term is negligible the dynamics is ruled by the Hartmann number $H$ only which is the ratio of the dissipation time to the Alfvén time, and scales like the inverse of the square root of the product of resistivity by viscosity. This occurs for the dynamics of the RFP as seen by 3D numerical simulation. When $H$ is small two basins of SH are shown to coexist. In the vicinity of $H=2000$ the system displays a temporal intermittency whose laminar phases are of QSH type. For higher $H$ 's the system reaches a multiple helicity $(\mathrm{MH})$ state whose features, in particular magnetic chaos, are analogous to the traditional turbulent state of RFP plasmas [18].

The resilience to chaotic perturbations of a one-parameter one-degree-of-freedom Hamiltonian dynamics is shown to increase when its corresponding separatrix vanishes due to a saddle-node bifurcation. This is important for the magnetic chaos of QSH states of the RFP. It brings a rationale for the confinement improvement of helical domains experimentally found for QSH plasmas; such a feature would not be expected from the classical resonance overlap picture as the separatrix disappearance occurs when the amplitude of the dominant mode increases [17]. 


\section{Active rotation of MHD modes}

The experiments on the active control of the MHD modes with a rotating external perturbation [19], have been extensively pursued and the physical mechanism of mode interaction with external fields has been analysed. This allowed us to develop an operational mode to obtain the active rotation of the locked dynamo modes (LDM) through the current flat-top phase (see Figure 3).

The active rotation of the modes is obtained with the Rotating Toroidal Field Modulation (RTFM) technique [19], through a current modulation in the 12 sectors of the toroidal winding. In this way a rotating toroidally localised $m=0$ perturbation of the toroidal field is applied to the plasma. The rotating perturbation is most effective if its rotation frequencies is $\omega<35 \mathrm{~Hz}$ and its toroidal mode content is mainly $n=1$. When continuous rotation sets in, the LDM rotates with a toroidal velocity equal to the driving perturbation and the latter leads the LDM by an angle $<\pi / 2$. This behaviour can be explained by the interaction of the external field with the tearing modes. The basic interaction is between the external $m=0$ perturbation with the internally resonant $m=0$ mode. Then the effects are transferred to the $m=1$ internally resonant modes thanks to the non-linear 3-wave interaction mechanism which underlies the LDM [20].

The external $m=0$ field interacts with the plasma inducing a sheet current at the resonant surface (the field reversal surface $r=r_{0}$ ), The current is proportional to the radial component of the external field $B_{\mathrm{r}}{ }^{0,1}\left(\mathrm{r}_{0}\right)$. In this way an electromagnetic torque is exerted on the $m=0$ mode given by:

$$
T_{e x t z}^{0,1} \propto b_{r}^{0,1} B_{r}^{0,1}\left(r_{0}\right) \sin (\Delta \phi)
$$

where only the toroidal component $T_{\text {extz }}^{0,1}$ is considered, because of the strong damping of the poloidal rotation in a torus. In order to induce rotation of the LDM, $T_{\text {extz }}{ }^{0,1}$ must by high enough to overcome the braking torque on the $m=0$ mode due to static error fields. Once that is true, the $m=0$ mode can hook up to the applied perturbation. To this end a balance must be reached between the drag torque due to plasma viscosity and eddy currents in the vessel [21] (both 
proportional to $\omega$ ) and $T_{\text {extz }}{ }^{0,1}$. This means that $\Delta \phi$ saturates at a value in the interval $0 \div \pi / 2$, i.e. in the stable range where the applied torque is an increasing function of $\Delta \phi$.

The applied torque $T_{\text {extz }}{ }^{0,1}$ is transferred to the $m=1$ modes via the non-linear coupling. For any couple of $m=1$ modes with toroidal number $n$ and $n+1$, the torques due to 3-wave interaction with the $m=0$ mode is given by [22]:

$$
\begin{aligned}
& T_{z}^{0,1} \propto C_{n} \sin \left(\phi^{1, n+1}-\phi^{1, n}-\phi^{0,1}\right)=C_{n} \sin \left(\Delta \phi^{3 w}\right) \\
& T_{z}^{1, n} \propto n C_{n} \sin \left(\phi^{1, n+1}-\phi^{1, n}-\phi^{0,1}\right)=n C_{n} \sin \left(\Delta \phi^{3 w}\right) \\
& T_{z}^{1, n+1} \propto-(n+1) C_{n} \sin \left(\phi^{1, n+1}-\phi^{1, n}-\phi^{0,1}\right)=-(n+1) C_{n} \sin \left(\Delta \phi^{3 w}\right)
\end{aligned}
$$

where $C_{n}$ is a coefficient proportional to the amplitude of the modes and to their overlap integral and $\phi^{\mathrm{m}, \mathrm{n}}$ is the phase of the $(m, n)$ mode. It is seen that the torque on the $m=1$ modes is larger than that on the $m=0$ by a factor $\mathrm{n}$ (with $\mathrm{n}>7$ for the typical spectrum in RFX). Such a leverage effect is responsible for the fact that the EM torque given by (2) is strong enough to maintain the phase locking of all of the $m=1$ modes (i.e. $\Delta \phi^{3 \mathrm{w}} \approx$ const) both in standard pulses and in those with active mode rotation. In the latter cases the external perturbation induces a linear rate of change of $\phi^{0,1}$, hence the phase locking condition requires either $\phi^{1, n}$ or $\phi^{1, n+1}$ to follow the rotation: it is a dynamic equilibrium where the action of $T_{\text {extz }}^{0,1}$ rotates both the $\mathrm{m}=0$ mode and one of the $m=1$ modes (the one subject to less braking torque). The other $m=1$ mode remains stationary because of the phase locking condition which makes $\Delta \phi^{3 \mathrm{w}}$ saturate at a value such that the torque given by (2) is just sufficient to match the braking torque on the rotating $m=1$ mode. Iterating the scheme for all of the $n, n+1$ couples of modes one sees that the $m=1$ mode subject to the highest braking torque can never be hooked up by the perturbation, hence its phase remains stationary. Such mode is typically the one with the largest amplitude, e.g. the $n=9$ one in Figure 4. The condition $\Delta \phi^{3 \mathrm{w}} \approx$ const implies

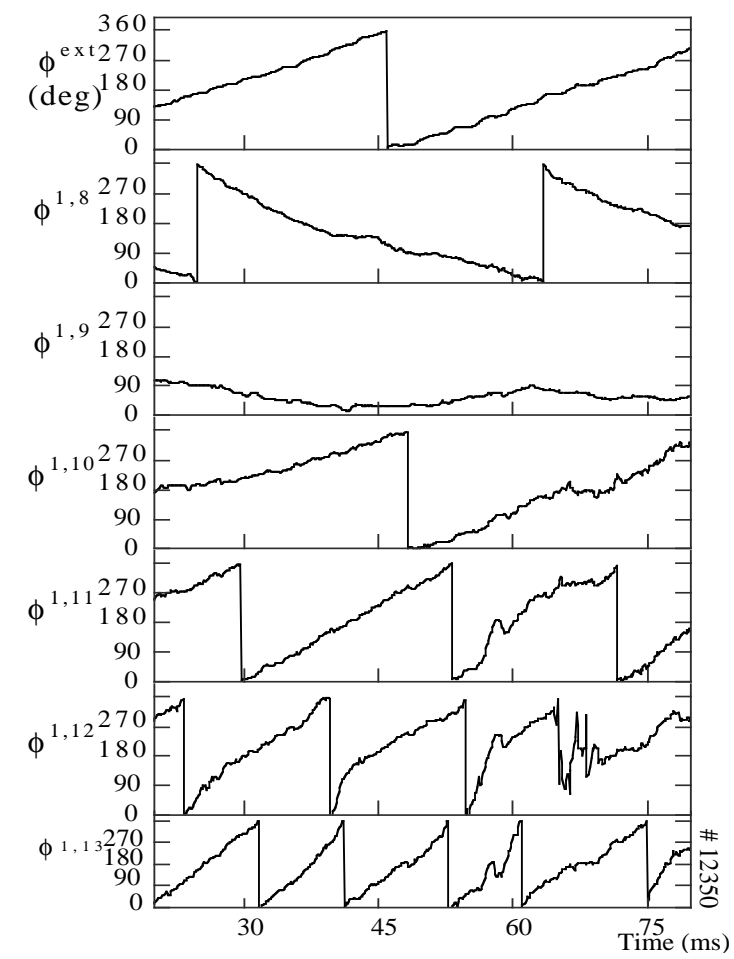

Figure 4: A pulse with continuous mode rotation through the current flat-top phase. Note the phase delay $<\pi / 2$ as long as rotation goes on. Note also the $(1,8)$ mode travelling backward and the rotation frequency increasing with $n$ for $n>10$ 
also that the mode with $n=n_{\text {stationary }}+1$ must co-rotate with the external perturbation, whereas the one with $n=n_{\text {stationary }}-1$ has to go backward. Moreover, the next adjacent $m=1$ mode $\left(n=n_{\text {stationary }}\right.$ +2 ) must rotate relative to the $n=n_{\text {stationary }}+1$, i.e. it moves at twice $\omega$ in the laboratory frame; and so on for modes with higher or lower $n$. All the above features are found in the example shown in Figure 4, which is a remarkable evidence of the non-linear coupling underlying the LDM.

The recipe to obtain mode rotation through the current flat top entails enforcing a toroidal position of the LDM during the current rise phase by applying a stationary $m=0$ perturbation, which acts as seed for the mode locking. Then the RTFM is applied with an initial phase which satisfies the $\Delta \phi<\pi / 2$ condition. The reliable rotation of the locked modes constitutes a major step toward routine operation at high currents in RFX, with good control of particle recycling and of plasma density [23]. Carbon blooming, a typical consequence of former high current operation, is avoided by spreading the heat load on the first wall. The driving field required for rotation increases with plasma current and density, and with rotation frequency. The last relationship is readily understood in terms of the linear dependence of the drag torque on $\omega$. The density dependence is probably due to the associated effect on the temperature, hence on the drag torque linked to plasma viscosity. Finally, the current scaling could be due to the decrease of the amplitude of the $m=0$ modes which show a scaling stronger than the $m=1$ ones. With the present power supplies, a rotation frequency of $20 \mathrm{~Hz}$ is obtained up to the density limit for current below the MA level, whereas in discharges at $1 \div 1.2 \mathrm{MA}$ active mode rotation is only possible at $10 \mathrm{~Hz}$ and with density corresponding to an $\mathrm{I} / \mathrm{N}$ parameter of 3 .

\section{Conclusions and future directions}

Recent experimental and theoretical studies have allowed a deeper insight into the MHD properties of the RFP configuration. A set of successful experiments has demonstrated the possibility of influencing both the amplitude and the spectrum of the magnetic fluctuations which characterise the RFP configuration. With these studies a reduction of the magnetic chaos has been obtained. The continuos rotation of wall locked resistive tearing modes has been obtained by an $m=0$ rotating perturbation. This perturbation induces rotation of $m=1$ nonlinearly coupled modes. Besides important information on the non-linear origin of the torque acting on the internally resonant modes, this rotation experiment proves the possibility of high current RFP operation without severe plasma-wall interaction. A way to a stationary reduction of magnetic chaos has been indicated also by the discovery of experimental QSH states, where a better confined helical core is produced. The simultaneous exploitation of several techniques for edge and core magnetic stochasticity reduction appears therefore promising for the future of the RFP configuration [23].

In the future the issue of stability, accessibility and robustness of Single Helicity states should be theoretically addressed by incorporating new elements: shell radius larger than plasma radius, heat transport (filamentation effects might be present), role of the pinch parameter and of the aspect ratio. In particular linear stability theory should be developed for helically symmetric profile. Future work should be dedicated to assess the value of viscosity to be used in the Hartmann number so as to predict the scaling of this number towards more collisionless regimes. From the experimental standpoint, a wider field of analysis shall be opened by 
producing new harmonic magnetic fields in the outer plasma region, also by mean of feedback techniques. This has the goal to substantially improving the capability of interacting with MHD modes. Results on the dependence of the $m=1$ modes dynamics on the aspect ratio $[12,15]$ suggest that a low R/a RFP might be promising in terms of achievement of QSH states and of active mode control, as less $m=1$ modes are present into the plasma in this condition. These studies might also show an increasing correlation of the low $R / a$ RFP physics with that of other compact devices, like spherical torus or compact current-carrying helical systems.

\section{References}

1 ROSTAGNI, G., Fus. Eng. Design 25, 301, (1995).

2 ORTOLANI, S. and SCHNACK, D.D. Magnetohydrodynamics of Plasma Relaxation., World Scientific, Singapore (1993).

3 CARAMANA E.J., et al., Phys. Fluids 26, 1305 (1983)

4 KUSANO K., SATO T., Nucl. Fusion 27, 821 (1987).

5 CAPPELLO S., BISKAMP D., Nucl. Fus. 36, 571 (1996).

6 PRAGER S.C., Plasma Phys. Contr. Fusion 41, A129 (1999)

7 GARZOTTI L., et al., Phys. Rev. Lett., 84, 5532, (2000).

8 TERRANOVA D., et al. Plasma Phys. Contr. Fus., 42, 843 (2000)

9 VALISA M., et al., J. Nucl. Mater. 241-243, 988, (1997).

10 MARTIN P, Plasma Phys. Contr. Fusion 41, A247 (1999).

11 ESCANDE D.F. et al., Phys. Rev. Lett. 85, 1662 (2000).

12 MARTIN P., et al., Phys. Plasmas 7, 1984 (2000)

13 TAYLOR J. B., Phys. Rev. Lett., 33, 1139 (1974).

14 ESCANDE D.F., et al., "The RFP as a magnetically quiet and non chaotic configuration", this conference (Paper TH3/4) (2000).

15 HO Y.L. et al., Phys. Plasmas 2, 3407 (1995).

16 SPIZZO G., et al., "Mitigation of plasma wall intercation during QSH states in RFX", to be published in Jou. Nucl. Materials (2000).

17 ESCANDE D.F., et al., "Chaos healing by separatrix disappearance and QSH states of the RFP", to be published in Phys. Rev. Lett. (2000).

18 CAPPELLO S., ESCANDE D.F., "Bifurcation in viscoresistive MHD: the Hartmann number and the RFP", to be published in Phys. Rev. Lett. (2000).

19 BARTIROMO R., et al., Nucl. Fusion 39, 1697 (1999).

20 FITZPATRICK R., Phys. Plasmas 6, 1830 (1999).

21 FITZPATRICK R., et al. Phys. Plasmas 6, 3878 (1999).

22 HEGNA C.C., Phys. Plasmas 3, 4646 (1996).

23 ANTONI V., et al., "Transport mechanisms and enhanced confinement in RFX", this conference (Paper EXP5/09) (2000). 